\title{
Una aproximación a los retos de la integración centroamericana. La perspectiva de docentes en tres universidades de la región"
}

José J. Romero Rodríguez SJ

Fundación ETEA para el Desarrollo y la Cooperación. Correo electrónico: jjromero@etea.com

LAS TRES UNIVERSIDADES JESUITAS DE CENTROAMÉRICA (Universidad José Simeón Cañas - UCA de El Salvador, Universidad Centroamericana - UCA de Nicaragua y Universidad Rafael Landívar de Guatemala) han iniciado un acción conjunta interdisciplinar e interuniversitaria sobre el tema específico de la integración centroamericana como marco para el desarrollo de la región. En ese contexto se han celebrado en las tres universidades sendas jornadas de estudio y reflexión destinadas a docentes sobre dicho tema monográfico. El presente estudio resume los resultados de los trabajos en grupo acerca de los problemas principales de los países, de cómo la integración puede ayudar a resolverlos y sobre cuáles son los aspectos críticos del propio proceso de integración desde la perspectiva de los participantes. El trabajo realizado hasta ahora ha desembocado en la formulación de un programa regional de investigación y formación de postgrado. El artículo concluye precisamente con un avance de la ruta crítica a seguir para este abordaje académico del tema.

Palabras clave: Centroamérica / integración centroamericana / desarrollo / universidades jesuitas / investigación

\section{Introducción}

La integración centroamericana atraviesa una coyuntura a la vez dinámica y problemática, "confusa pero vibrante" (Caldentey del Pozo, 2010, cap.7). Para bien y para mal está de actualidad y es objeto de análisis y debates contradictorios. Algunos autores se han esforzado por definir cuáles son los retos principales del proceso,

Como se explica más adelante, este texto -que se publica simultáneamente en las revistas Encuentro de la UCA de Nicaragua y Estudios Centroamericanos de la UCA de El Salvador- es fruto del proceso de formulación del "Proyecto de investigación interuniversitaria regional sobre la situación y perspectiva del proceso de integración centroamericana como marco para el desarrollo de la región", presentado al Programa de Cooperación Interuniversitaria e Investigación $(\mathrm{PCl})$ de la Agencia Española de Cooperación Internacional al Desarrollo. La resolución de aprobación del proyecto fue publicada con posterioridad en el BOE no 14 de 16 de enero de 2010 (p. 3945; código A/024221/09). 
confiando en abrir de esa forma vías para su reactivación, bajo el supuesto de que la integración constituye un marco más adecuado para el desarrollo de la región (véase entre otros: Caldentey del Pozo, 2010; Solís, 2009; Granados, Lizano \& Ocampo, 2008; Osterlof Obregón, 2008 y Prado, 2008. Sobre la problemática regional, véase Programa Estado de la Nación, 2008). Según esas visiones, no se trataría de pedirle a la integración la solución para todos los problemas centroamericanos, sino de determinar aquellas áreas de "bienes públicos regionales", siguiendo una denominación actual, que podrían ser abordadas por la institucionalidad regional de cara a la mejora de las condiciones de vida de los habitantes de la región. Son precisamente esos retos, debidamente priorizados y aplicando rigurosamente el principio de subsidiariedad, los que deberían servir de base para la definición de una agenda "factible" (Caldentey del Pozo, 2010) ${ }^{1}$ que, superando la retórica y el maximalismo endémicos en los políticos del área, devuelva al proceso centroamericano de integración una legitimidad en buena parte perdida.

El presente texto pretende aportar alguna luz sobre dichos retos mediante la sistematización del pensamiento de una cualificada muestra de docentes universitarios de las tres universidades centroamericanas confiadas a la Compañía de Jesús. Aun siendo conscientes de las limitaciones metodológicas del estudio que presentamos, creemos que constituye un aporte significativo al debate antes aludido. Lo publicamos confiados en que pueda contribuir a suscitar una mayor conciencia de la realidad y perspectivas del citado proceso de integración en el seno de la Academia.

En efecto, con honrosas excepciones, es conocida la ausencia del tema de la integración de los programas y de la agenda de preocupaciones de las universidades centroamericanas en general, y de las confiadas a la Compañía de Jesús en particular. Aparte de otras razones, como pueden ser la desacreditación mediática del proceso, el desprestigio derivado del mal funcionamiento de algunas instituciones o de la mala imagen de algunos de sus líderes, una razón primaria es la ignorancia que existe sobre el tema entre los propios docentes e investigadores. Con algunas honrosas excepciones, no han existido programas específicos significativos de formación ni se han desarrollado prácticamente investigaciones sobre esta materia.

Esta escasez de formación e investigación en materia de integración regional en las universidades centroamericanas durante décadas ha tenido impactos negativos en la propia integración centroamericana. En las instituciones de la región hay funcionarios buenos y menos buenos -como en toda institución, incluidos los gobiernos nacionales. Pero no existe lo que se denomina una "comunidad epistémica" acerca de esta materia. Precisamente, la falta de referentes teóricos explica la dificultad que gobiernos e instituciones han tenido para defender el proyecto de integración, complejo y de largo plazo, frente a estrategias alternativas como las derivadas de los acuerdos de libre comercio que tienen un impacto más inmediato pero menos estructural y profundo.

La universidad es seguramente la instancia más adecuada para profundizar y extender este debate. La formación e investigación en materia de integración regional es indispensable para que actores políticos, sociales y económicos la aborden con el detalle necesario. Cada vez más prioridades en las políticas de desarrollo de los países de la región están 
relacionadas con el ámbito regional y es, por tanto, preciso disponer de herramientas y conocimientos de análisis sobre el proceso de integración.

Por otro lado, las convocatorias de selección de personal ligadas a la integración se encuentran con dificultades para cumplimentar los criterios sobre formación, experiencia y conocimientos de los procesos de integración. Asimismo, aunque hay un grupo de investigadores y consultores cada vez más centrados en este tema, hay una presencia excesivamente elevada de consultores internacionales o de consultores con conocimiento muy superficial en los concursos convocados desde gobiernos, instituciones y cooperación internacional. La universidad tendría la misión de proporcionar investigaciones e investigadores que puedan aportar rigor a estos estudios y proyectos, así como una reflexión profunda y sostenida sobre las implicaciones de la integración para los retos del desarrollo regional y sobre las contribuciones que pudiera hacer para responder a ellos.

La preocupación por colaborar en llenar este vacío ha sido el motivo que ha llevado a las tres universidades jesuitas centroamericanas a plantearse la conveniencia de promover un programa conjunto de investigación y de formación de postgrado acerca de la integración centroamericana. El trabajo que aquí presentamos es el resultado de las actividades iniciales de dicha iniciativa; confiamos que será seguido por otros productos, resultados de investigaciones concretas interuniversitarias e interdisciplinarias.

\section{Contexto y marco de la investigación}

Esta investigación ha tenido lugar en el marco de una serie de tres jornadas de formación y reflexión sobre Integración Centroamericana realizadas en 2009 los días 24 y 25 de agosto en la Universidad Rafael Landívar (URL) en la Ciudad de Guatemala, 31 de agosto y 1 de septiembre en la Universidad Centroamericana (UCA) en Managua, 3 y 4 de septiembre en la Universidad Centroamericana (UCA) José Simeón Cañas en San Salvador.

La actividad forma parte de un proyecto conjunto de formación y sensibilización de las tres universidades jesuitas centroamericanas citadas para atender a la integración regional como tema de reflexión, docencia e investigación. Se aspira a converger en un programa común de postgrado y de investigación de alcance regional sobre el tema de la integración centroamericana ${ }^{2}$. De esta forma se refuerza también la consecución del objetivo estratégico de colaboración entre las tres universidades jesuitas de la región, por el que se viene trabajando con especial intensidad en los últimos años. La iniciativa cuenta con el apoyo técnico de la Fundación ETEA para el Desarrollo y la Cooperación de Córdoba (España), con una larga tradición de más de dos décadas de colaboración en la región, con un énfasis especial en los estudios de integración y teniendo como contrapartes privilegiadas precisamente a estas universidades.

La actividad ha estado dirigida a docentes de estas universidades. Las Jornadas han estado promovidas por un equipo interuniversitario formado por las profesoras Renata Rodrigues (Vice-rectora académica de la UCA de Nicaragua), Lucrecia Méndez (entonces Directora de posgrado y hoy vice-rectora académica de la URL) y Lidia Salamanca (Directora de postgrado de la UCA de El Salvador). La organización recayó en un equipo formado por Luis 
Alberto Lemus (URL), Mauro Ampié (UCA - Nicaragua) y Sergio Bran (UCA - El Salvador), apoyados por personas de los equipos de sus facultades o departamentos respectivos y de la Fundación ETEA ${ }^{3}$.

\section{Metodología}

El colectivo consultado estuvo formado por unos 60 docentes de las tres universidades, alrededor de 20 docentes por universidad. Las especialidades dominantes entre los participantes eran: Derecho, Economía, Ciencias Políticas, Sociología, Administración de Empresas y Comunicación. Se trata, por tanto, de una muestra cualificada, pluridisciplinar, que -aunque obviamente no es representativa de la población total- aporta información muy significativa acerca de las opiniones de estudiosos de las Ciencias Sociales sobre los problemas de los países en donde se ubican sus respectivas universidades y acerca de las potencialidades y los retos del proceso de la integración centroamericana para afrontarlos.

En las tres universidades los participantes respondieron, en sendas sesiones de trabajo en grupo, a dos cuestionarios idénticos. No se trata, por tanto, de una encuesta individual, sino de la respuesta cualificada de un total de nueve grupos de cuatro a cinco docentes cada uno, que se construyó sobre la base de la discusión, debate y búsqueda de consenso. Podríamos calificar a estos grupos como "multidisciplinarios", "especializados" y "focales", en la medida que estaban constituidos -como ya se ha dicho- por profesores universitarios de diversas áreas científicas. 
El cuestionario sobre el que se trabajó fue el siguiente:

Primera sesión de trabajo en grupo

Objetivo: Facilitar el intercambio de reflexiones entre los participantes sobre la situación, perspectiva y retos de la integración centroamericana y su repercusión en el país respectivo (Guatemala, Nicaragua, El Salvador)

Preguntas:

1 ¿Cuáles son los 3 problemas principales de nuestro país?

2 ¿Qué y cómo puede aportar la integración a esos problemas detectados?

3 ¿Cuáles son los aspectos críticos de la integración en la actualidad?

Segunda sesión de trabajo en grupo

Objetivo: Aportar ideas y sugerencias de cara a la formulación del programa interuniversitario de investigación y formación sobre la integración centroamericana a desarrollar por las universidades jesuitas centroamericanas

Preguntas:

1 ¿Cuáles serían los temas de investigación relacionados con la integración centroamericana susceptibles de ser incluidos en el programa interuniversitario de las universidades jesuitas centroamericanas?

2 ¿Cuáles serían las características fundamentales de un programa de formación en integración centroamericana auspiciado por las universidades jesuitas centroamericanas? A continuación sugerimos el siguiente esquema de apoyo: Formato (doctorado, postgrado, maestría etc.; presencial, semipresencial, virtual etc.); contenidos básicos; público objetivo; otros aspectos.

En los tres apartados siguientes pretendemos sistematizar los resultados más relevantes de dichos trabajos en grupo en lo relativo a la primera sesión de trabajo. El primer eje de análisis presenta las semejanzas o concordancias a las que llegaron las tres universidades respecto al estado actual de la integración centroamericana, con su respectivo análisis de los retos que se le plantea y de sus implicaciones académicas (apartado 4). El apartado 5 aborda las diferencias por país, y propone algunas reflexiones sobre las implicaciones derivadas de la diversidad de contextos nacionales y académicos. Por un último, en un apartado 6 conclusivo se presenta la ruta crítica que se ha diseñado a fin de que en estas instituciones académicas se aborde de manera activa la integración como marco de desarrollo para Centroamérica.

\section{Principales resultados. Primer eje: concordancias entre los países}

\subsection{Respecto a los problemas de cada país}

Comenzaremos reflejando la existencia de una coincidencia significativa en el diagnóstico de los problemas prioritarios que aquejan a los tres países analizados (Guatemala, Nicaragua y El Salvador). Hay tres grupos de problemas que son priorizados por todos los grupos con expresiones similares, aunque con la lógica diversidad: a) la falta de gobernanza y la fragilidad institucional; b) los aspectos relativos a la pobreza, la exclusión y la desigualdad; 
c) los problemas de inseguridad ciudadana y delincuencia. Aunque las formulaciones varían ligeramente, la coincidencia es grande.

a) Problemas relacionados con la falta de gobernanza y la fragilidad institucional

- Debilidad de las instituciones, con falta de representatividad, derivada de la polarización social y política

- Falta de participación de las minorías políticas (jóvenes, grupos étnicos, mujeres)

- Falta de políticas de Estado

- Falta de confianza ciudadana en las instituciones

- Falta de respeto al Estado de Derecho: falta participación ciudadana, auditoría social y existe corrupción en todos los niveles

- Fragilidad institucional: falta de obligatoriedad de la ley, el Estado es visto como un botín

- Inestabilidad política

- Desequilibrio exacerbado del poder

b) Problemas relacionados con pobreza, exclusión, desigualdad

- Pobreza

- Desempleo

- Inequidad y exclusión

- Desigualdad socioeconómica

c) Problemas de inseguridad

- Violencia

- Inseguridad ciudadana

\subsection{Sobre el aporte de la integración centroamericana a la solución de esos problemas}

Algunos grupos de participantes no han sido suficientemente sistemáticos a la hora de sugerir medidas que se podrían tomar en el ámbito de la integración para luchar contra los problemas comunes reseñados en el apartado 4.14. En todo caso, refiriéndonos, a los tres grandes ámbitos señalados en el apartado anterior, en los que existía clara convergencia a nivel de diagnóstico, estas son algunas sugerencias de algunos:

a) Frente a los problemas relacionados con la falta de gobernanza y la fragilidad institucional

- La integración permitirá posicionar la ciudadanía centroamericana (y asegurar la protección de los derechos de manera regional).

- La integración debe ser una política de cada país, estatal; esto generaría la coincidencia entre fortalecimiento institucional y toma de decisiones, lo cual debe generar la negociación como bloque.

- Para eso se necesita reforzar las instituciones y los procesos que apunten a otras debilidades como la débil democratización.

- En resumen: fortalecer la institucionalidad nacional fortaleciendo la regional. 
b) Frente a los problemas de pobreza, exclusión y desigualdad

- Hay que buscar políticas que favorezcan de manera clara a los sectores y a los países menos privilegiados, con la conciencia de un fortalecimiento regional.

- Sería un espacio de articulación de políticas públicas regionales de cara a la reducción de la exclusión socio-económica.

c) Frente a los problemas de inseguridad

- Es fundamental la formulación de políticas públicas a escala regional para afrontar el narcotráfico, el tráfico de armas, la trata y la migración. Es fundamental pensar una legislación conjunta, manejo y circulación de la información (bases de datos comunes etc.).

- En base al fortalecimiento institucional propuesto, se generaría una mejora en el funcionamiento de las instituciones. La corrupción, los intereses políticos y la presencia de poderes ocultos generan influencia directa en la debilidad de las instituciones.

- La integración y el interés por fortalecer y crear políticas de Estado son el camino para mejorar estos aspectos. Y al revés, a más empoderamiento ciudadano sobre los beneficios de la integración, mayor sostenibilidad de la misma.

- El regional sería un espacio de articulación de políticas públicas regionales para atender la prevención y combate de la violencia, etc.

\subsection{Sobre los aspectos críticos de la integración}

A la hora de realizar el diagnóstico sobre dichos aspectos críticos, los grupos de las tres universidades coincidieron en señalar una serie de ámbitos, aunque lo expresen de formas diferentes ${ }^{5}$.

\section{Aspectos relacionados con la debilidad de la institucionalidad centroamericana}

- Falta de institucionalidad de las políticas de integración.

- Falta de capacidad supranacional de las instituciones regionales.

- Problemas de inoperancia de la institucionalidad regional.

- Falta de credibilidad de las instituciones.

- Necesidad de reforzar las instituciones de integración.

- Desgaste y desprestigio de las instituciones regionales, como el PARLACEN.

- Falta de coherencia entre las decisiones tomadas por los órganos decisorios del SICA con la capacidad de ejecución del Sistema.

Aspectos relativos a la falta de voluntad política para avanzar en la integración

- Visión cortoplacista de los gobiernos; falta de visión política.

- Ausencia de políticas vinculadas a la integración en las agendas de los partidos.

- La integración no es una política de Estado.

- Falta de compromiso de los países en el proceso.

- Los esfuerzos de integración son transitorios: no hay seguimiento.

- Falta de interés de la clase política.

- Falta de visión de los políticos centroamericanos. 
Aspectos relacionados con el incumplimiento de los acuerdos

- Divorcio entre lo real y lo formal.

- Desfase entre los instrumentos jurídicos firmados y su ejecución.

- Muchos instrumentos que no se cumplen.

- Falta de cumplimiento de los acuerdos.

- Negociación por separado de acuerdos de libre comercio.

- No todos los países están incorporados al mismo nivel en el proceso.

Aspectos relacionados con la escasa participación social en la integración

- Hace falta más voluntad y participación de todos los sectores.

- Es preciso involucrar a las instituciones y a la sociedad civil.

- El proceso excluye a los actores sociales.

- Desconocimiento del proceso de integración.

- Déficit de apertura a la participación de la sociedad civil.

- Faltan canales de participación social en el proceso.

- Falta de información sobre el proceso, ausencia de consultas populares, déficit de legitimidad.

- Falta una visión progresista, adoptando mecanismos de información y difusión que reflejen los beneficios que trae consigo la integración.

- Falta fortalecer la visión centroamericana.

En conjunto estas aportaciones dibujan un diagnóstico sumamente adecuado de la situación, coincidiendo claramente con otros analistas del proceso de integración.

\section{Principales resultados. Segundo eje: diferencias según países}

Como era de esperar, las respuestas a los cuestionarios -además de las coincidencias señaladas en el apartado anterior- han puesto de manifiesto algunas diferencias resultantes de los diferentes contextos nacionales, tanto en lo relativo a la problemática de cada país susceptible de beneficiarse de un tratamiento integrado, de índole regional, como a las expectativas y a la valoración que la propia integración les merece.

\subsection{Sobre los problemas de cada país}

Comenzando por los problemas, predominan, como ya se ha indicado en el apartado 4.1, las coincidencias en el diagnóstico. Pero si se analizan atentamente las respuestas a los cuestionarios se observan algunos matices significativos, que se explican por las peculiaridades propias del contexto de cada uno de los tres países.

\section{Guatemala}

Los universitarios guatemaltecos destacan tres problemas específicos de su país que no fueron recogidos como tales por las otras dos universidades:

- Inseguridad alimentaria que provoca deterioro en el medio ambiente y afecta al mercado interno. 
- Déficit del mercado interno: menos consumo, menos demanda; ello afecta a la educación, la salud y la infraestructura.

- Polarización y falta de consenso a nivel nacional: diferencias sociales, de clases e ideológicas.

\section{Nicaragua}

- Llama la atención que no aparezca entre los problemas destacados la inseguridad ciudadana, claramente presente en los otros dos países. Ello parece responder a una percepción objetiva sobre este país.

- Tres de los cuatro grupos destacan un rasgo específico de la problemática nicaragüense, formulado concretamente como la "falta de una visión de nación".

- Un grupo se refiere al sistema de la estructura económica que no aporta mayor valor agregado y no fomenta la producción industrial.

- Otro grupo menciona los problemas económicos locales presupuestarios.

\section{El Salvador}

- $\quad$ En el caso de El Salvador existe una clara coincidencia entre las prioridades de los tres grupos de trabajo. Varios concuerdan con las destacadas por los docentes de las otras dos universidades, pero aparece una especial preocupación por los problemas medioambientales que no surgió en los anteriores.

- Por último, uno de los grupos prioriza el déficit de identidad de la realidad salvadoreña.

\subsection{Sobre el aporte de la integración centroamericana a la solución de esos problemas}

Los universitarios de cada país formulan de la siguiente forma el aporte de la integración a los problemas específicos que destacaron (además de los comunes a todas las universidades vistos más arriba, en el apartado 4.2).

\section{Guatemala}

- Frente a la inseguridad alimentaria: la integración puede ayudar a generar política ambiental común de producción y de ambiente, aranceles comunes, para un beneficio a largo plazo.

- Frente al déficit del mercado interno: la integración puede ayudar en el sentido de establecer normas de transparencia sobre el gasto público. Asimismo, el Gobierno debe determinar políticas públicas, la descentralización de la actividad económica, del poder y de los servicios públicos.

- Frente a la polarización y falta de consenso nacional: la búsqueda de consenso en los actuales órganos de la integración, especialmente el PARLACEN, puede influir en las instituciones nacionales a través de los partidos políticos representados en ambos escenarios, de tal manera que ayude a resolver el problema de la debilidad institucional. Es decir, que estos partidos políticos pueden coadyuvar a fortalecer a las instituciones nacionales para que adquieran mayor grado de representatividad de los intereses nacionales. 


\section{Nicaragua}

- En relación a la "falta de una visión de nación", hay algunas alusiones indirectas: a) la integración podría ayudar (desde fuera) a fortalecer el Estado de Derecho y los derechos humanos en el interior del país; b) de forma parecida, se indica que el fortalecimiento de las instituciones regionales contribuiría al fortalecimiento de la institucionalidad en Nicaragua; c) asimismo piensan que ello ayudaría a disciplinar y fortalecer a la sociedad y a recuperar la credibilidad de las instituciones.

- En cuanto al fomento de la actividad económica, los docentes nicaragüenses indican que un mercado único más amplio, de casi 40 millones de personas -con libre movilidad de factores, lo que favorecería la inversión y la generación de empleo, el aumento del consumo etc.- ayudaría a dinamizar la economía de la región y a reducir el desempleo.

- El grupo que menciona los problemas económicos locales parece querer sugerir que la integración podría ayudar (también desde fuera) a aplicar mejor la subsidiariedad con políticas estatales que favorezcan a los gobiernos locales.

\section{El Salvador}

- Sobre los problemas medioambientales, se piensa que la integración regional puede convertirse en un espacio de articulación de políticas públicas regionales para luchar contra el deterioro y la vulnerabilidad ambiental.

- Encuanto al déficit de identidad de la realidad salvadoreña, no aparece explícitamente en las respuestas de los grupos cómo la integración puede ayudar a superarlo; implícitamente puede encontrarse en el siguiente párrafo.

- Sí hacen un aporte interesante, típicamente salvadoreño, y es que primero hay que resolver los problemas a nivel interno del país (políticas fiscales, atención a los sectores más desfavorecidos, rendición de cuentas, fortalecimiento de las instituciones, mecanismos de regulación etc.) antes de pensar en procesos de integración.

- $\quad$ En ese sentido, subrayan especialmente la urgencia de un pacto fiscal, social, como apuesta por el Estado del bienestar que permita luego la integración.

\subsection{Sobre los aspectos críticos de la integración}

En este apartado, con ser muchos los aspectos en que coinciden los grupos de las tres universidades -como se ha visto en el apartado 4.3- hay sin embargo algunos aportes específicos de cada país que seguramente podrán ser explicados a partir del contexto nacional. Pero tampoco hay que desechar la hipótesis de que la selección depende de la perspectiva individual y/o de la disciplina de los propios componentes de los grupos universitarios.

\section{Guatemala}

- Necesidad de mantener el principio de solidaridad y de estabilidad democrática (caso Honduras).

- Capital humano débil.

- Las economías centroamericanas no son complementarias, sino competitivas. 


\section{Nicaragua}

- Rechazo por el gobierno de Nicaragua a lo que se considera una cesión de soberanía en beneficio de las instituciones de la integración.

- $\quad$ No se vincula la integración, ni el quehacer de sus instituciones, con las necesidades más urgentes de la población.

\section{El Salvador}

El grupo de este país es el que presenta más aspectos no citados por los colegas de las otras dos universidades. Evidentemente, no son cuestiones totalmente desligadas, pues en esta materia todo está relacionado con todo. Reproducimos a continuación estos ítems específicos:

- Falta de rendición de cuentas.

- Existencia en Centroamérica de una identidad y visión del mundo más fragmentada que integrada.

- Discurso nacionalista que busca diferenciarse del "otro" de manera radical.

- Existencia del crimen organizado que sabe que en la medida en que la región esté dividida pueden ellos mantener sus propios procesos paralelos de "integración".

- Dependencia de la cooperación internacional.

- Mentalidad inadecuada de los funcionarios del SICA.

- Debilidad en la realización de un pacto social centroamericano. Se ha priorizado el eje económico-comercial.

\subsection{Algunas propuestas sugeridas para mejorar algunos aspectos críticos citados}

Como vimos, hay un relativo consenso a la hora de diagnosticar los aspectos críticos de la integración (cfr. Apartado 4.3). Varios grupos participantes proponen algunas medidas tendentes a luchar contra algunos aspectos críticos -particularmente en el ámbito de la participación social- pero no se ha hecho de forma sistemática. Las ubicamos en esta sección 5 , relativa a las diferencias, porque no se trata de propuestas en que exista concordancia entre los grupos de docentes de las distintas universidades.

\section{Aspectos relacionados con la escasa participación social en la integración}

- Someter a consultas populares las decisiones estratégicas sobre la integración. Abrir canales de participación social en el proceso de integración (Guatemala).

- Realizar campañas de información y promoción de debates sobre el tema, en todos los ambientes sociales (Guatemala).

- Reconocimiento de la juventud y de minorías políticas como sujetos de derecho y de generación de empleo (Guatemala).

- Legitimar los procesos sobre la base de participación, consulta y decisión de la ciudadanía: "construir legitimidad" (Guatemala).

- Fortalecimiento de los actores socio-políticos diversos, dando prioridad a los sectores o grupos excluidos (El Salvador).

- Fomentar la integración en los movimientos de base, de la ciudadanía. Favorecer una integración ciudadana (El Salvador). 


\section{A modo de conclusión: una primera ruta para el abordaje por la academia del tema de la integración}

Las jornadas han permitido a) incrementar el conocimiento y el interés académico de docentes de las tres universidades por el tema de la integración centroamericana; b) detectar en cada universidad a las personas más interesadas en esta cuestión y su disponibilidad para acometer proyectos de más envergadura al respecto tanto en el ámbito de la investigación como de la formación; c) definir las temáticas más relevantes que podrían ser objeto de investigación en la fase siguiente y los intereses de cada país al respecto.

A partir de las ideas recibidas en los trabajos grupales de las tres jornadas, una comisión interuniversitaria se reunió el 7 de septiembre en la UCA de San Salvador con el fin de formular un proyecto conjunto de investigación que fue presentado a la convocatoria 2009 del Programa de Cooperación Interuniversitaria de la AECID, siendo la entidad solicitante ETEA (Córdoba) en el marco de la Universidad de Córdoba, de la cual es centro adscrito. Las tres universidades han decidido poner en marcha un proyecto conjunto de acuerdo a los objetivos que aparecen a continuación.

\section{Objetivo general:}

Reforzar las instituciones y estructuras que soportan el sistema de ciencia, tecnología, e innovación de varias universidades centroamericanas de prestigio, a ser posible una por país, mediante la constitución de una Red Interuniversitaria Centroamericana estable de cooperación científica y de investigación, que desarrollará un Programa a mediano plazo de Investigación Regional sobre Integración Centroamericana y Desarrollo. El núcleo inicial del programa estará formado por las citadas tres universidades confiadas a la Compañía de Jesús.

\section{Objetivos específicos:}

- Constituir y/o consolidar una red con la conformación de un equipo inter-universitario y multidisciplinario.

- Desarrollar investigaciones concretas sobre ejes claves para analizar el estado de avance de la Integración Centroamericana.

- Formular recomendaciones para los gobiernos y organismos de integración.

- Formular un proyecto de postgrado que incluirá un plan de estudios y malla curricular que permita la incidencia académica en el proceso de integración centroamericana

Las líneas de investigación seleccionadas en principio han sido las siguientes:

1. Enfoque jurídico-institucional de la integración: fundamentos jurídicos de la integración.

2. Aspectos económicos - comerciales de la integración - comercial (una exploración del comercio intrarregional).

3. Enfoques nacionales sobre la integración centroamericana y su repercusión en las políticas de integración. (¿Qué pretenden los gobiernos con la integración? ¿Qué no 
deben pretender los gobiernos con la integración centroamericana?).

4. Integración social (retos sociales de la integración, integración e inclusión cultural, representatividad de los diferentes sectores en el marco de la integración centroamericana, participación ciudadana, espacios de discusión en materia de integración, etc.).

5. Participación ciudadana y de la sociedad civil en su diversidad en el proceso de integración.

Para cumplir estos objetivos se ha diseñado un primer plan de acción ${ }^{6}$ que consiste en sendos trabajos de estudio e investigación en cada universidad, a cargo de pequeños equipos seleccionados adecuadamente, completados con encuentros y talleres interuniversitarios. Reproducimos a continuación las actividades previstas y su diseño temporal ${ }^{7}$.

Actividad 0: investigación en su sede universitaria (mes 1-12).

Actividad 1: $1^{\circ}$ taller (centroamericano). Objetivo: designar las líneas de investigación temática, designación de la dirección de las mismas y de los equipos integrantes. Fecha: Mes 1. Duración: 2-3 días.

Actividad 2 a 5: 4 encuentros temáticos locales (en cada universidad). Meses 2, 4, 6, 8. Además, a lo largo de todo el período se podrán celebrar talleres virtuales, aprovechando las potencialidades ya existentes de conexión virtual de las universidades.

\section{Actividad 6 a 8:}

$2^{\circ}$ taller (hispano-centroamericano). Objetivos: avances de los contenidos de las líneas de investigación, unificación de criterios, bibliografía, metodología, reparto de tareas y planificación del calendario restante del programa. Fecha: Mes 3-4. Duración: 2-3 días.

$3^{\circ}$ taller centroamericano. Objetivos: avance en las líneas de investigación, unificación de criterios, comunicación con el equipo español y planificación del resto del programa. Fecha: Mes 6-7. Duración: 2-3 días.

$4^{\circ}$ taller centroamericano. Objetivo: propuestas de conclusiones de líneas de investigación, unificación de criterios, comunicación con el equipo español y planificación del resto programa. Fecha: mes 8-9. Duración: 2-3 días.

Actividad 9: $5^{\circ}$ taller y seminario de cierre (hispano-centroamericano). Objetivo: presentación de conclusiones y conferencias de expertos sobre las líneas de investigación elegidas; comunicación y difusión de resultados a: rectores, comunidad universitaria, Secretaría General del SICA, gobiernos, opinión pública; decisión sobre publicación. Duración: 3-5 días.

En consecuencia, esta es la relación de productos esperados de esta primera etapa de trabajo en común: 
- Consolidación de la propia red interuniversitaria.

- Realización de algunas investigaciones preliminares sobre el estado de avance de los principales retos de la integración centroamericana en relación con el desarrollo de la región. Estarán articuladas en torno a los ejes de investigación y se basarán principalmente en fuentes secundarias: investigaciones documentales, talleres de discusión, entrevistas focalizadas, métodos de investigación cualitativa.

- Formulación del Programa de investigación a medio plazo sobre Integración Regional y desarrollo articulado por ejes de investigación.

- Formulación del Programa conjunto de postgrado en integración regional centroamericana y desarrollo para presentar a alguna fuente de financiación.

- Elaboración de alguna(s) publicación(es).

- Formulación de recomendaciones a los gobiernos y/o la institucionalidad del SICA

Por último, la estructura organizativa del grupo interuniversitario sería la siguiente:

- Coordinación a cargo de una persona responsable por las universidades centroamericanas y otra por ETEA.

- Consejo Académico-Administrativo: formado por las directoras de postgrado de las universidades participantes.

- Comisiones interuniversitarias por eje de trabajo. Participarán unos tres docentes por cada universidad.

\section{Agradecimientos}

Esta publicación no hubiera sido posible sin el apoyo a todo el proyecto de las autoridades responsables de las tres universidades mencionadas y sin la eficaz actuación del equipo responsable de la organización, personas todas mencionadas nominalmente en el apartado 2 del texto. El autor agradece también las sugerencias aportadas por Sergio Bran (UCA de El Salvador) a una versión preliminar del texto. Recibiremos complacidos las sugerencias de nuestros lectores.-

\section{Notas}

1 En otras ocasiones el mismo autor ha abogado por una agenda "racional y contenida".

2 El desencadenante concreto de este proyecto ha sido la presentación del mismo a la convocatoria del Programa de Cooperación Interuniversitaria de la Agencia Española de Cooperación Internacional al Desarrollo (AECID), en su convocatoria 2009.

3 Al final de las jornadas, los participantes respondieron a un detallado cuestionario de evaluación. Es significativo el alto grado de satisfacción por el trabajo realizado que se reflejó en dicha evaluación: la valoración global de las jornadas, medida en una escala de 1 a 5 fue la siguiente: UCA de El Salvador: 4,3; URL de Guatemala: 4,93; UCA de Nicaragua: 4,94. La evaluación del trabajo de los grupos (cuyos productos son objeto de este artículo) fue la siguiente: UCA de EI Salvador: 4,4; URL de Guatemala: 4,53; UCA de Nicaragua: 4,59.

4 Ello puede deberse a una falta de claridad en la formulación de esta pregunta en el cuestionario.

5 Las repeticiones corresponden a formulaciones parecidas de los distintos grupos de docentes.

6 Reproducimos la propuesta tal como ha sido presentada a la convocatoria del Programa de Cooperación Interuniversitaria de la AECID. Naturalmente, en función de las circunstancias se podrán producir modulaciones del plan de trabajo inicialmente 
previsto.

7 Los meses empiezan a contar a partir de la aprobación del programa por la AECID.

\section{Referencias bibliográficas}

Caldentey del Pozo, P. (2010). Panorama de la integración centroamericana: dinámica, intereses y actores. En P. Caldentey \& J. J. Romero (Eds.) El SICA y la UE: la integración regional en una perspectiva comparada. San Salvador: Programa de Formación en integración regional para funcionarios centroamericanos, Fundación ETEA para el Desarrollo y la Cooperación.

Caldentey del Pozo, P. \& Romero, J. J. (Eds.). (2009). El SICA y la UE: un análisis de integración regional comparada. San Salvador: Ed. Fundación ETEA y UCA editores (en prensa).

Granados, J., Lizano, E. \& Ocampo, F. (2008). El desafío de un acuerdo de asociación entre Centroamérica y la Unión Europea. San José: Academia de Centroamérica.

Osterlof Obregón, D. (Ed.). (2008). América Latina y la Unión Europea: una integración esperanzadora pero esquiva. San José de Costa Rica: Facultad Latinoamericana de Ciencias Sociales (FLACSO).

Prado, M. (Ed.). (2008). Alianza estratégica entre Centroamérica y Europa. Más allá de un acuerdo. San José de Costa Rica: Centro Internacional para el Desarrollo Humano.

Programa Estado de la Nación (Costa Rica). (2008). Estado de la Región en Desarrollo Humano Sostenible. Un informe desde Centroamérica y para Centroamérica / Programa Estado de la Nación. San José de Costa Rica: Estado de la Nación.

Solís, L. G. (2010). Las agendas regionales de desarrollo. En P. Caldentey del Pozo \& J. J. Romero (Eds.). El SICA y la UE: la integración regional en una perspectiva comparada. San Salvador: Programa de Formación en integración regional para funcionarios centroamericanos, Fundación ETEA para el Desarrollo y la Cooperación. 